\title{
Contribuições à Identificação de Distúrbios de Qualidade de Energia com uso da Transformada Wavelet
}

\author{
Amanda T. S. Monteiro* Yago B. Moreira* \\ Mateus de M. Araújo* Francisco V. E. Lemos* \\ Pablo R. Lopes* Aryfrance R. Almeida* \\ * Programa de Pós-Graduação em Engenharia Elétrica, Universidade \\ Federaldo Piauí, (e-mail: amathayla@hotmail.com, \\ mademeloa@outlook.com,yagomoreira@gmail.com, \\ victorleteves@gmail.com,prlopes4@yahoo.com.br, \\ aryfrance@ufpi.edu.br).
}

\begin{abstract}
The Wavelet transform is commonly used in power systems as a mechanism capable of detecting and locating the beginning, end, and duration moments of the disturbances and faults present in the signals. In this paper, an algorithm developed in the MATLAB software was implemented, which detects these faults through multiresolution analysis by prior determination of a wavelet family. The results obtained in the simulations prove the effectiveness of the proposed methodology always determining the start and end instants with good precision in the majority of the analyzed cases.

Resumo: A transformada Wavelet é comumente empregada em sistemas de potência como um mecanismo capaz de detectar e localizar os instantes de início, fim, e duração dos distúrbios e falhas presentes nos sinais. Com isso, no presente artigo implementou-se um algoritmo desenvolvido no software MATLAB, que detecta estas falhas através da análise de multirresolução pela determinação prévia de uma família de wavelet's. Os resultados obtidos nas simulações comprovam a eficácia da metodologia proposta sempre determinando os instantes de início e fim com boa precisão na maioria dos casos analisados.
\end{abstract}

Keywords: Electrical Disturbance; Identification of disturbances; Multiresolution; Signals; Wavelet Transform

Palavras-chaves: Distúrbios elétricos; Identificação de distúrbios; Multirresolução; Sinais;Transformada Wavelet.

\section{INTRODUÇÃO}

O sistema elétrico de potência (SEP) está sujeito a diversos tipos de distúrbios elétricos, que são classificados como: transitórios, variações de tensão de curta duração ou sustentadas, desequilíbrios, distorções na forma da onda, flutuação ou ainda variações na frequência do sistema elétrico. Estes distúrbios influenciam nos indicadores da Qualidade de Energia Elétrica (QEE) (Deckmann and Pomilio, 2011), prejudicando diretamente os equipamentos ligados ao SEP.

Neste contexto, é necessário uma avaliação dos distúrbios recorrentes no SEP. Para tal, é comum a utilização de técnicas de análise de sinais. O trabalho de (Weeks, 2010) utiliza a técnica de transformada discreta de Fourier, para análise de sinais em que as componentes de frequência são invariáveis no tempo. .

Entretanto, a transformada de Fourier tem limitações na análise de sinais com transitórios devido a escolha da largura das janelas, responsável pela resolução no tempo e frequência do espectro, pois quando a largura é ajustada para uma boa resolução no tempo, há uma perda na resolução de frequência e o inverso também ocorre. Para observação de sinais elétricos é necessário a análise do sinal em um plano de múltiplas escalas, com larguras de tempo e banda de frequência variando de acordo com o valor da frequência central de banda.

Assim, diante das limitações citadas, a Transformada Wavelet pode ser utilizada empregando funções de análise que são locais tanto no tempo, quanto em frequência (Wilkinson and Cox, 1996).

O presente trabalho propõe utilizar a transformada wavelet, em conjunto com a transformada discreta de Fourier para determinar o instante de inicio e término de distúrbios de qualidade de energia. O desempenho visto no trabalho Kern (2008) encontra-se de acordo com a teoria, transformada de Fourier concomitantemente com a transformada Wavelet facilita a obtenção dos instantes de distúrbios.

A seção seguinte deste documento, é apresentado uma forma geral da precisão da transformada wavelet para solucionar distúrbios recorrentes no Sistema Elétrico de Potência. Na terceira seção é exposto o procedimento realizado para obtenção da localização dos distúrbios e seus res- 
pectivos tempos de duração. Finalmente, na quarta seção são apresentados os resultados obtidos com as simulações realizadas no software MATLAB, afim de comprovar a metodologia implementada.

\section{TRANSFORMADA WAVELET APLICADA A QUALIDADE DE ENERGIA ELÉTRICA}

A transformada de wavelet é uma ferramenta matemática para análise de sinais no domínio do tempo e frequência que a partir de uma função base, conhecida como wavelet mãe, origina novas funções com escalas diferentes. A wavelet também conhecida como wavelet mãe $\phi(\mathrm{t})$ e a função de escala $\theta(\mathrm{t})$ são as componentes bases dessa transformada. A função de escala comprime a wavelet mãe em uma escala de 2, múltiplas vezes, enquanto apresenta um atraso de $\mathrm{k}$. Assim, novas funções são originadas a partir da wavelet mãe, com novas escalas construídas como tal (Ali and Sharif (2018)).

A Transformada Discreta da Wavelet (Discrete Wavelet Transform - DWT), definida em (1), proporciona uma descrição mais eficiente, pois são transladadas e escalonadas em intervalos discretos.

$$
D W T(m, d)=\int_{-\infty}^{\infty} f(t) \psi_{m, d}(t) d t
$$

Desta forma, são úteis na detecção e extração de características de perturbações de distúrbios, pois é sensível para sinalizar irregularidades, mas insensível à regularidade do comportamento do sinal. A DWT é fruto do refinamento da transformada wavelet, e caracteriza-se por Filtros de diferentes frequências de corte, para analisar o sinal em diferentes escalas (Ray et al. (2010)).

A transformada wavelet constitui um poderoso método para um diagnóstico mais preciso do regime de sistemas de distribuição, como relatado no trabalho de (Pereira (2002)), facilitando a detecção de distúrbios. O que permite ser implementada em diferentes trabalhos, no qual, apenas a técnica utilizada para detectar e localizar as pertubações inseridas no sistema pode ser alterada. No trabalho de (Delmont et al. (2003)), o estudo foi elaborado com base na aplicação da Análise Multirresolução, que detecta e localiza distúrbios agregados às formas de ondas de tensão, apresentando resultados satisfatórios.

A análise multirresolução consiste em analisar sinais em múltiplas bandas de frequência, permitindo a implementação da transformada wavelet discreta, através de sucessivo agrupamento de filtro formando pares: passa-baixa e passa-alta. Estes pares devem ser formados em cada estágio de escala da transformada wavelet. Portanto, como a análise multirresolução fornece soluções aos problemas em diferentes níveis, é utilizada para determinar as decomposições (ou detalhes) e a aproximação final do sinal com distúrbio.

Na Figura 1 é ilustrada a técnica da análise multirresolução em três níveis de resolução. Em que $\mathrm{f}(\mathrm{x})$ é o sinal original, $\mathrm{h}_{0}$ as aproximações, como os detalhes são obtidos por meio de bancos de filtros, que são formados por filtros passabaixa (h0) e passa-alta (h1). O filtro passa-alta tem a função de separar o conteúdo de alta frequência no sinal em análise, o filtro passa-baixa, por sua vez, remove os componentes de alta frequência (Kern (2008)).

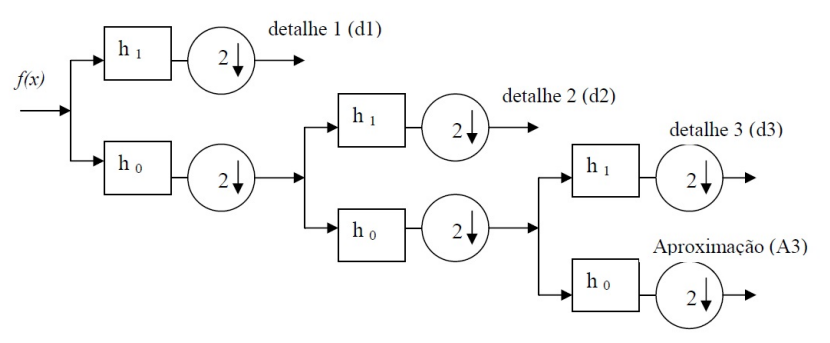

Figura 1. Processo de decomposição em Análise Multirresolução

Afim de facilitar a implementação e visando obter os instantes de início, fim e duração dos distúrbios, este trabalho propõe uma metodologia utilizando o ferramental da transformada wavelet para determinar estes parâmetros (Kern (2008)). A metodologia desenvolvida pode analisar sinais que apresentam um ou diversos distúrbios. Para detecção e localização no tempo é utilizado apenas a transformada wavelet. Para classificação das pertubações é necessário implementar técnicas distintas ,como análise através da Curva de Desvio Padrão e técnicas de inteligência artificial (Filho (2007)).

No software MATLAB, foi desenvolvido um algoritmo capaz de localizar, detectar e classificar os distúrbios presentes nos sinais, o procedimento realizado para a sua implementação é visto na seção seguinte.

\section{METODOLOGIA DESENVOLVIDA}

Inicialmente foi gerado um sinal periódico por meio da função seno, esse sinal foi denominado sinal sem distúrbio. Outro sinal, o sinal com distúrbio, foi gerado modificando a amplitude do sinal sem distúrbio em determinado período de tempo. Portanto os dois sinais possuíam a mesma fase, mas com diferença de amplitudes. Em cada um dos sinais foi realizado o processo de decomposição e reconstrução, referente ao processo de análise multirresolução.

Para o sinal com distúrbio, foi utilizado a transformada Wavelet para determinar as respectivas decomposições D1, D2, D3, D4, D5 e aproximação final do sinal. No MATLAB a Wavelet possui 21 tipos de famílias diferentes, dentre as quais destacam-se: Haar, Daubechies, Biorthogonal, Coiflets, Symlets, Morlet, Mexican Hat, Meyer.

Para cada tipo de sinal analisado é realizado uma escolha do tipo de Wavelet's mais adequada. Dentre os tipos de famílias diferentes de Wavelet's, é selecionada a que apresentar menor erro quadrático, erro medido entre a reconstrução do sinal (soma das decomposições e aproximação final) e o sinal original. Nessa escolha também está relacionada de forma intrínseca o tipo de distúrbio do sinal. Há família de Wavelet que é capaz de decompor com maior precisão distúrbios de alta frequência, por exemplo, ou aquelas que apresentam melhores resultados nos sistemas com harmônicas, subtensões ou interrupções, que são distúrbios recorrentes no sistema elétrico atual.

Após a definição do tipo de Wavelet e decomposição do sinal, calculou-se as Tranformadas de Fourier para as de- 
composições D1 a D5, para verificar o espectro de frequência de cada sub-elemento que compõe o sinal em análise. $\mathrm{O}$ procedimento de decomposição do sinal, determinação da família Wavelet e aplicação da transformada de Fourier é realizado em ambos sinais, com e sem distúrbio.

Para localizar as posições onde inicia-se e extingue-se as falhas, comparou-se as decomposições que apresentarem maior diferença entre os sinais com e sem distúrbio. Esta comparação é obtida por uso da correlação entre os sinais decompostos.

A correlação $(r)$ tem como principal finalidade comparar duas funções ou sinais $(u(k)$ e $y(k))$, com o intuito de avaliar sua similaridade, a fim de buscar uma dependência entre as mesmas, como em (2).

$$
r_{u y}(k)=\lim _{n \rightarrow \infty} \frac{1}{2 N+1} \sum_{i=-N}^{N} u(i) y(i+k)
$$

A partir da correlação obtém-se os sinais, dentre as decomposições e aproximações finais dos sinais com e sem distúrbio, que apresentam maiores diferenças. Colocando os sinais de forma sobreposta tem-se a localização e a duração do distúrbio detectado. A seção seguinte apresenta os resultados obtidos, que comprovam a metodologia proposta e implementada.

Para teste da metodologia, primeiramente adotou-se sinais com e sem distúrbios gerados a partir de uma função senoidal de mesma fase e amplitudes diferentes, simulando distúrbios de variação momentânea de tensão. Por fim utilizou-se um banco de dados do grupo IEEE P1159.3, de sinais com distúrbio (P1159.3 (2018)). Para cada sinal com distúrbio do banco de dados, gerou-se um sinal sem distúrbio de mesma fase que o sinal com distúrbio.

\section{RESULTADOS OBTIDOS}

As transformadas Wavelet e Fourier foram utilizadas neste trabalho para a análise de sinais elétricos, como distúrbios de qualidade de energia. A Wavelet é uma função capaz de decompor um sinal no domínio do tempo, em diferentes escalas no tempo e na frequência, através de sua transformada. Portanto, a localização e duração dos distúrbios foram realizados com essa função. Para determinar a amplitude do sinal utilizou-se a Transformada de Fourier. Um sinal originalmente no domínio tempo pode ser analisado em componentes de frequência através de Fourier. Inicialmente para confirmar o modelo, gerou-se uma senoide pura com seis períodos. A partir da mesma, um afundamento momentâneo de tensão de dois ciclos foi gerado, como é ilustrado na Figura 2.

Como mencionado anteriormente, o algoritmo desenvolvido determinou qual melhor família Wavelet, para esse caso a bior2.4 foi utilizada como mostrado na Tabela 1. Após a escolha da Wavelet pelo menor erro quadrático entre a reconstrução e o sinal original, a senoide original foi decomposta em escalas distintas e aproximação final como ilustrado na Figura 3 e as Transformadas de Fourier para cada decomposição e da aproximação são verificadas na Figura 4.

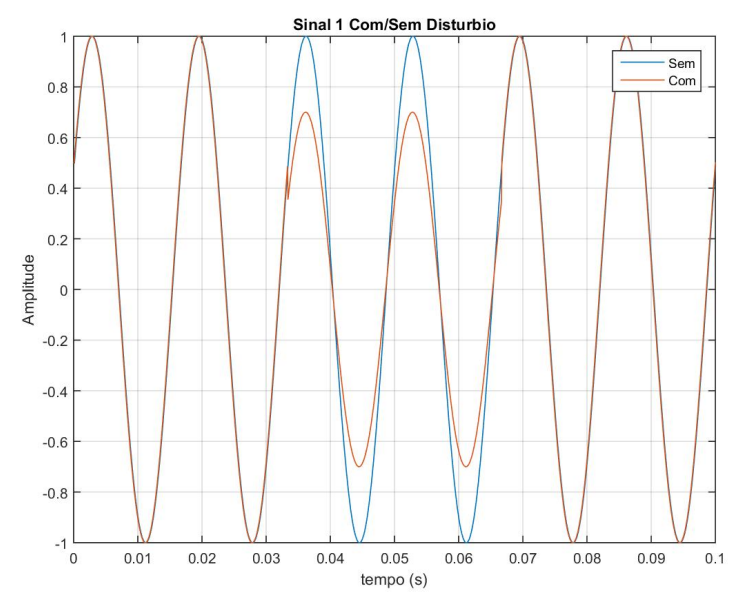

Figura 2. Exemplo de afundamento de tensão
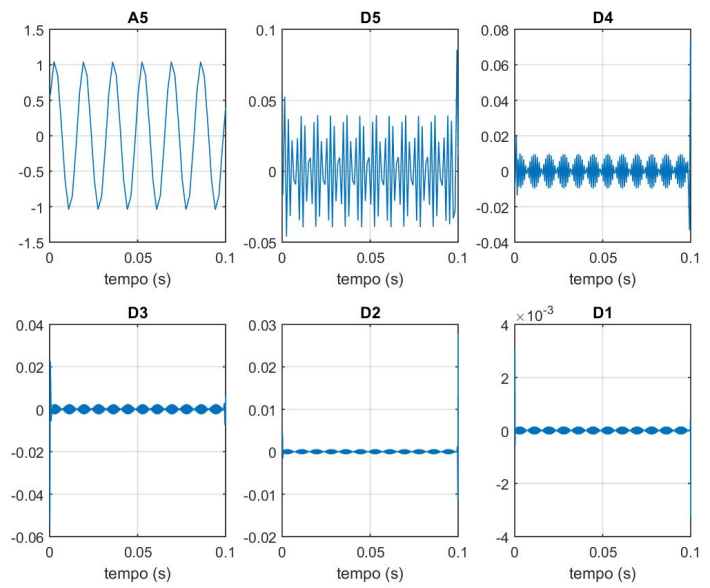

Figura 3. Decomposições e aproximação da Wavelet do sinal sem distúrbio
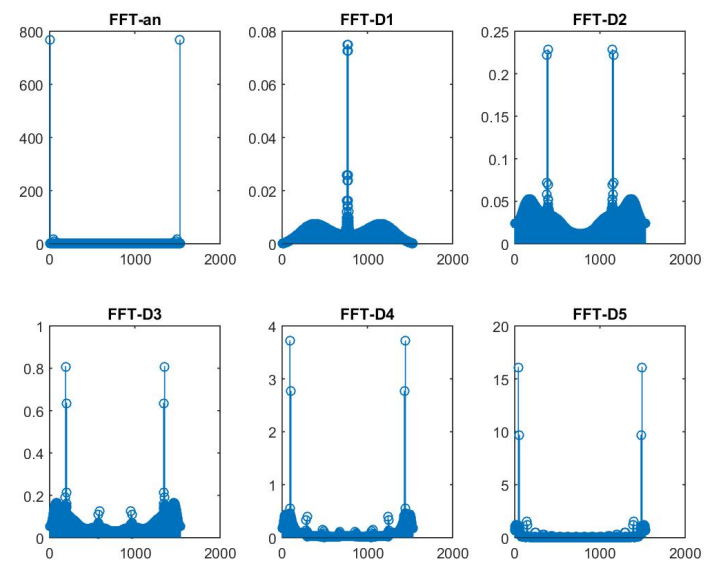

Figura 4. FFT das decomposições e aproximação da Wavelet do sinal sem distúrbio 

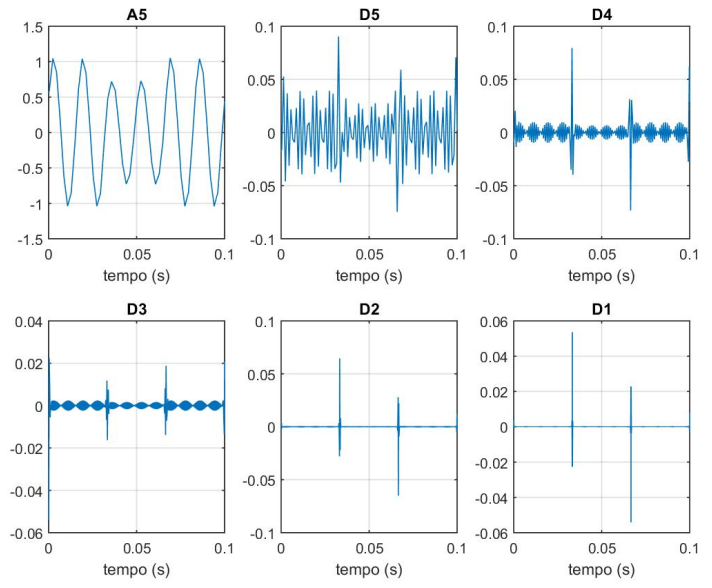

Figura 5. Decomposições e aproximação da Wavelet do sinal com distúrbio afundamento de tensão
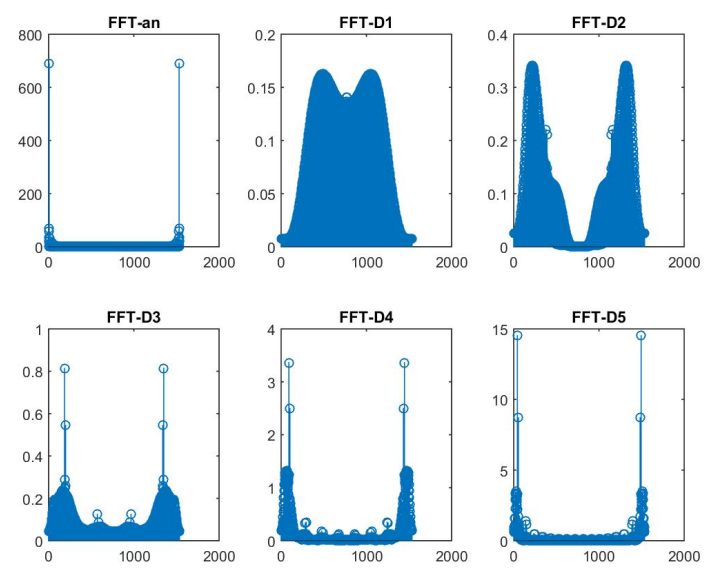

Figura 6. FFT das decomposições e aproximação da Wavelet do sinal com distúrbio afundamento de tensão

$\mathrm{O}$ procedimento anterior foi igualmente praticado para o sinal com afundamento de tensão. Novamente as decomposições e aproximação do sinal, bem como as componentes em frequência são representadas nas Figuras 5 e 6 .

Após a determinação dos detalhes e aproximações para cada sinal e de seus respectivos espectros em frequência foi realizada uma comparação entre os espectros dos detalhes correspondentes para os sinais sem distúrbio e com distúrbio. A figura de mérito empregada foi a correlação entre os sinais. Os espectros que apresentassem menor correlação determinariam que detalhes seriam utilizados para detecção do início e término do distúrbio. Para o sinal de afundamento momentâneo de tensão, o detalhe escolhido correspondeu a primeira dilatação da escala em frequência, que neste trabalho foi denominado "d1"para sinal sem distúrbio e "D1"com distúrbio para o sinal com afundamento, e outros que serão analisados mais adiante. A Tabela 1 resume as decomposições utilizadas para cada sinal, bem como a Wavelet escolhida.

Na Figura 7 estão representadas as decomposições para cada sinal, bem como a superposição entre elas. Analisando com mais precisão através da Figura 8 percebese que o início do afundamento foi próximo de $0.033 \mathrm{~s}$
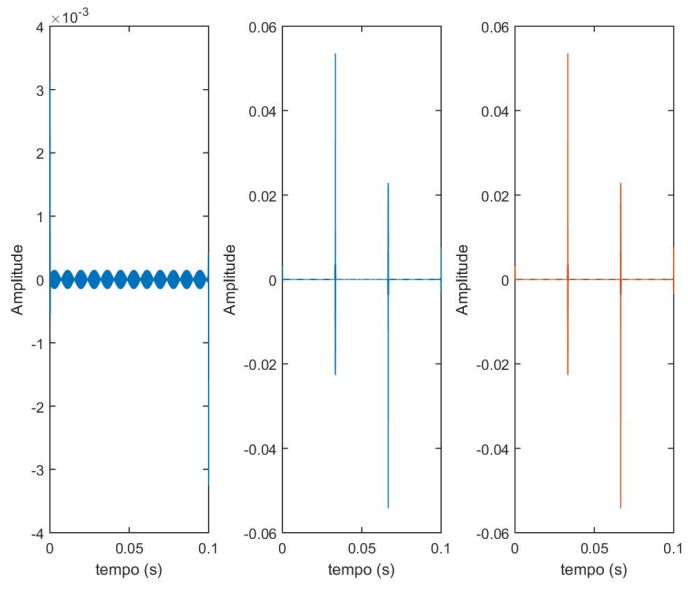

Figura 7. Decomposições escolhidas e localização do distúrbio

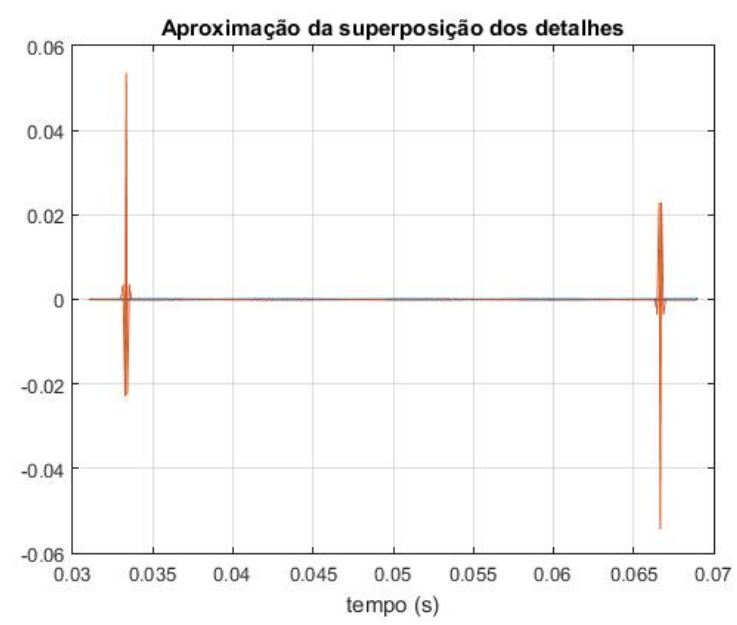

Figura 8. Aproximação das decomposições escolhidas

e o término em 0.067s. Depois da localização e duração do distúrbio, realizou-se a transformada de Fourier para o instante de duração do distúrbio em ambos os sinais. Verificando a Figura 9 concluiu-se que o distúrbio com duração próxima de 0,034 s foi um afundamento de tensão de 0,7 pu.

Para o sinal da Figura 10 repetiu-se os passos anteriores para escolha da Wavelet, da decomposição. As representações das decomposições, aproximações e espectros em frequência dos mesmos foram omitidos, restando apenas a análise das decomposições escolhidas e do espectro harmônico de cada sinal ilustrados nas Figuras 11 e 12.

Analisando as Figuras 11 e 12 percebe-se que ocorreu uma elevação momentânea de tensão de 1,3 pu com duração $0,05 \mathrm{~s}$.

Finalmente utilizou-se um sinal com distúrbio de um banco de dados do IEEE. Esse sinal pode ser verificado na Figura 13. Percebe-se que entre os instantes 0,02 e 0,04 segundos existe um transitório, e por toda onda ocorre uma defasagem em relação ao sinal sem distúrbio.

Na Figura 14 é exibida a comparação entre os detalhes, para determinação do instante de início e fim do distúr- 


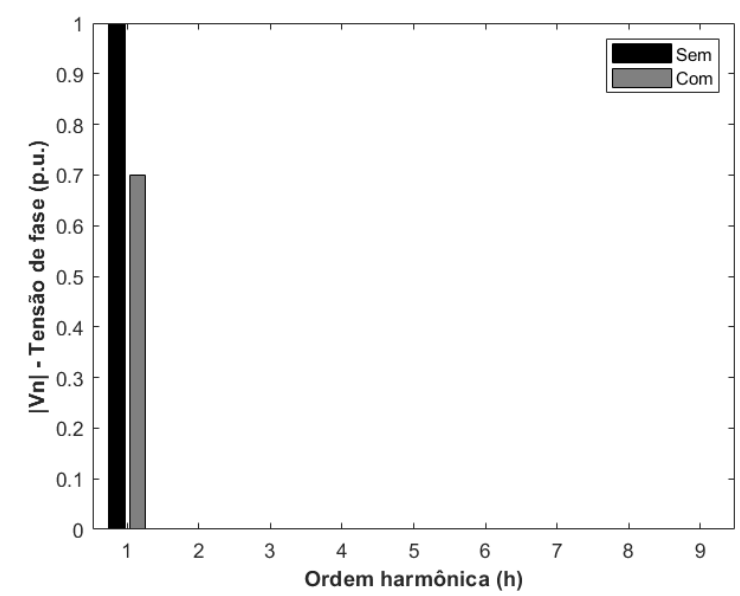

Figura 9. Componentes harmônicas do sinal com afundamento de tensão

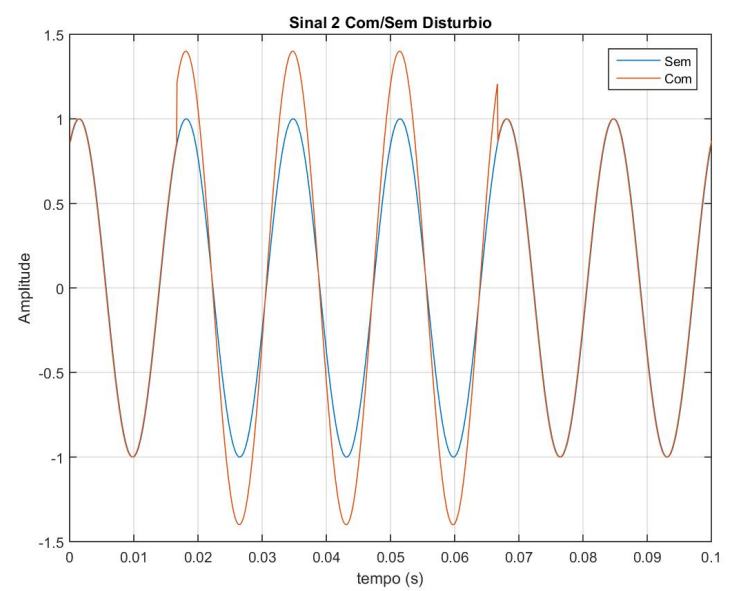

Figura 10. Exemplo de elevação de tensão

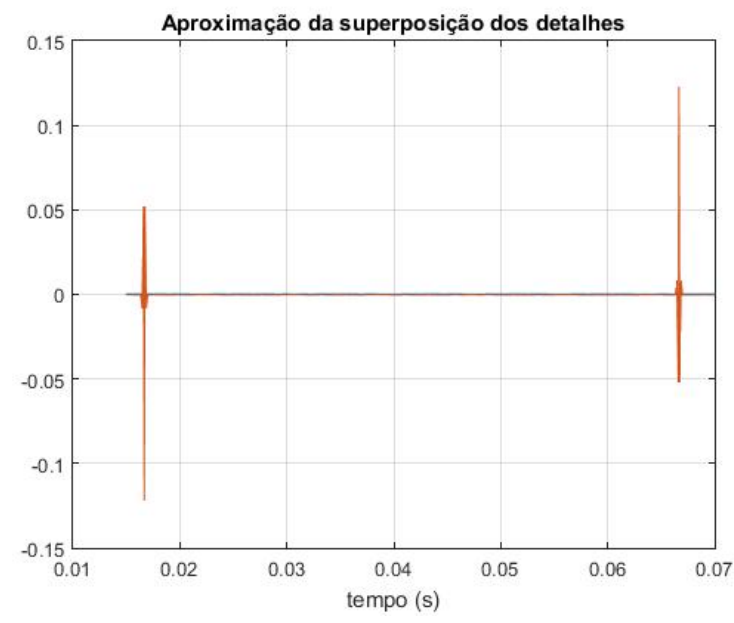

Figura 11. Aproximação das decomposições escolhidas

bio. A técnica para determinar a duração do distúrbio para esse caso mostra-se imprecisa, pois praticamente a decomposição da função com distúrbio sobrepõe a decomposição sem distúrbio. Isso acontece pois as duas ondas não estão sintonizadas corretamente. Para melhor determinar a duração do distúrbio, aproximou-se a superposição das

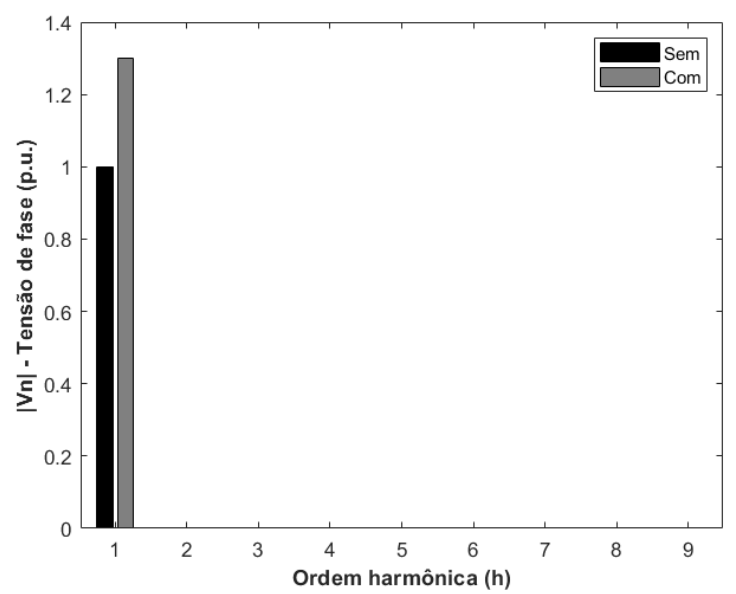

Figura 12. Componentes harmônicas do sinal com elevação de tensão

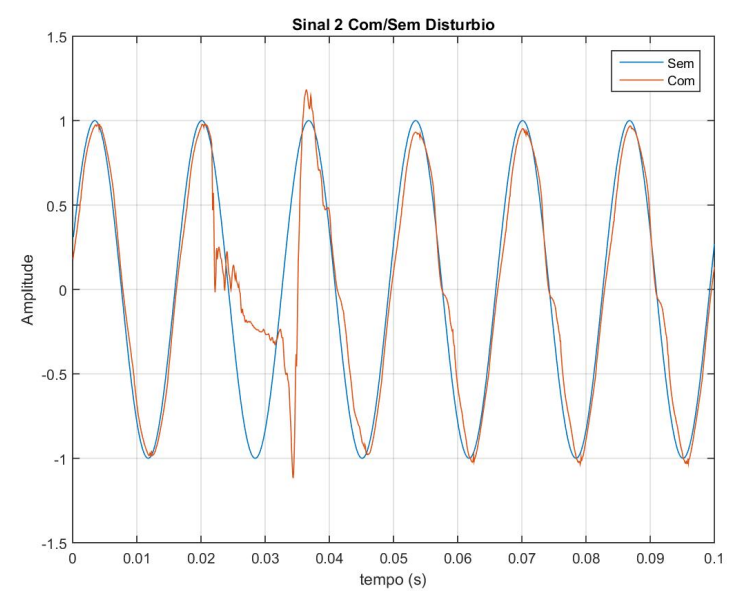

Figura 13. Sinal estudado
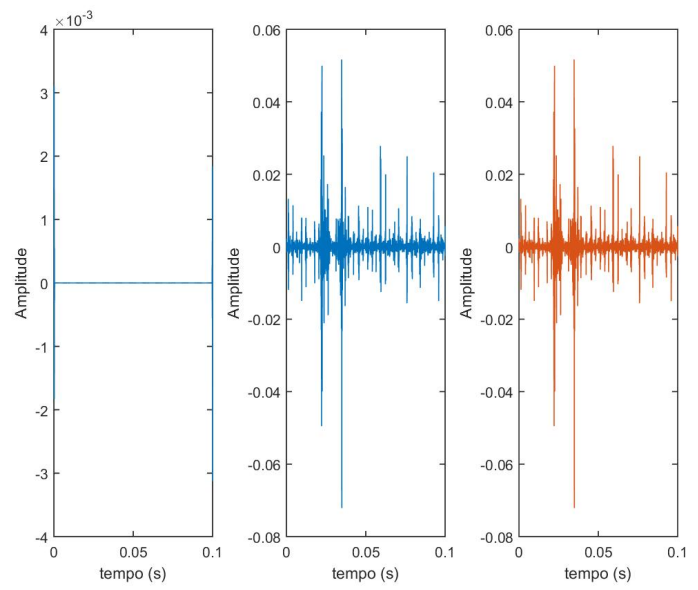

Figura 14. Decomposições e localização do distúrbio

decomposições, como pode ser verificado nas Figuras 15 e 16.

Através da Figura 15 denotou-se que o transitório presente no sinal do IEEE iniciou em 0,022 segundos e terminou em 


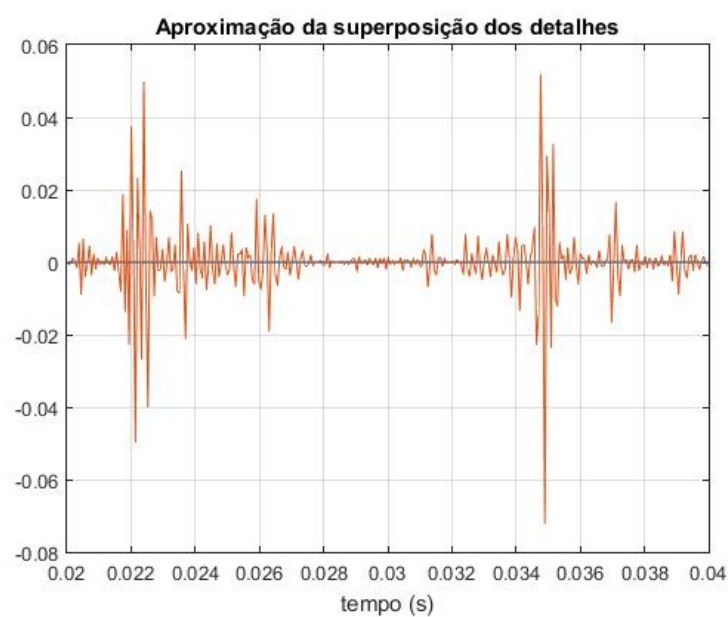

Figura 15. Aproximação das decomposições e localização do distúrbio

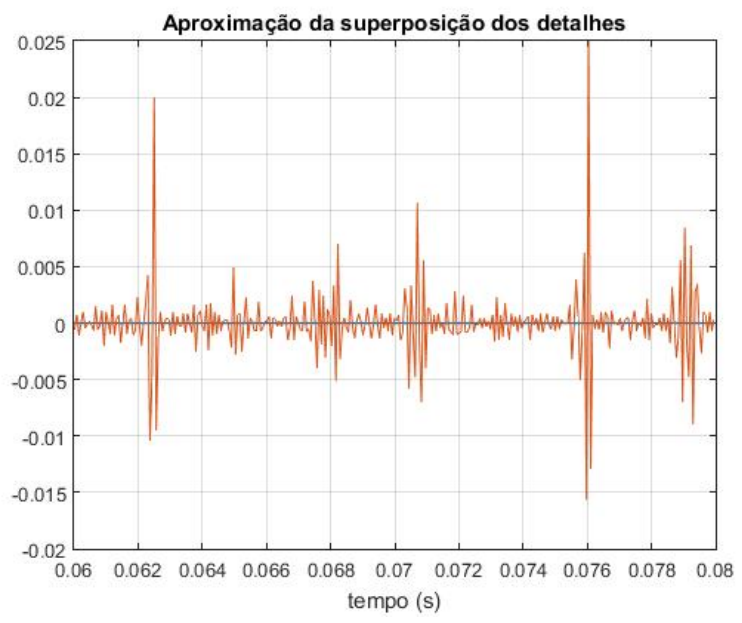

Figura 16. Aproximação das decomposições e localização do distúrbio

Tabela 1. Relação de Wavelets utilizadas para cada sinal

\begin{tabular}{ccccc} 
Sinal & Wavelet & Decomposições & Correlação & Erro \\
\hline Afundamento & bior2.4 & d1 e D1 & 0,2627 & $9,28 \mathrm{E}-32$ \\
Elevação & bior2.4 & d1 e D1 & 0,1795 & $6,65 \mathrm{E}-32$ \\
Distúrbio IEEE & rbio2.4 & d1 e D1 & 0,4805 & $9,41 \mathrm{E}-32$ \\
\hline
\end{tabular}

0,035 segundos, com base nos pontos de maior amplitude da decomposição.

Pela Figura 16 percebeu-se que nos instantes em que a amplitude da decomposição do sinal com distúrbio é muito maior que a amplitude da decomposição do sinal sem distúrbio, são tempos que ocorrem uma defasagem entre os sinais.

\section{CONCLUSÃO}

Neste trabalho foi desenvolvido uma metodologia de identificação de distúrbios em sinais. Esta metodologia se centra no uso da transformada de Wavelet com uso da Transformada de Fourier para determinar o período de inicio e termino do distúrbio, assim como duração.

Para os disturbios do tipo afundamento e elevação, a transformada de Wavelet mais adequada se mostrou como bior 2.4, enquanto para o Distúrbio IEEE, composto de vários sobrepostos, a transformada de wavelet mais adequada foi rbio2.4.

Durante a analise dos sinais com distúrbios, teve-se como problemática obter um perfeito casamento entre os sinais originais e um sem disturbios, uma vez que os primeiros não apresetavam qualquer informação de fases. Então para alguns sinais, devido a natureza dos disturbios relacionadas a elas, teve-se a incerteza do casamento entre fases dos sinais com e sem disturbios, que podem ter afetados certos resultados. Para este problema, busca-se novas soluções.

De forma geral, os resultados são muito animadores, uma vez que com uso de técnicas com um custo computacional relativamente baixas, permitiram caracterizar os instantes de tempos de diversos distúrbios. O que permite com que para trabalhos futuros integre-se a metodologia apresentada, alguma técnica de inteligencia computacional para caracterização dos distúrbios em analise.

\section{REFERÊECIAS}

Ali, H.H.S.M. and Sharif, S.M. (2018). Comparison between discrete wavelet transform and maximal overlap discrete wavelet transform as an analysis tool for h.264/avc video. In 2018 International Conference on Computer, Control, Electrical, and Electronics Engineering (ICCCEEE), 1-5. doi:10.1109/ICCCEEE.2018. 8515873.

Deckmann, S.M. and Pomilio, J.A. (2011). Avaliação da qualidade da energia elétrica. available in http://www.dsce.fee.unicamp.br/antenor/pdffiles/ qualidade/b5.pdf.

Delmont, O.F., Arruda, E., Oleskovicz, M., Carneiro, A.A.F.M., and Coury, D.V. (2003). Utilização da transformada wavelet e rnas para caracterização de distúrbios na qualidade da energia. Seminário Brasileiro sobre Qualidade da Energia Elétrica, 381-38.

Filho, O.D. (2007). Um algoritmo para detecção, localização e classificação de distúrbios na qualidade da energia elétrica utilizando a transformada wavelet.

Kern, F.G. (2008). Análise da qualidade de energia elétrica utilizando transformada wavelet.

P1159.3, I. (2018). IEEE P1159.3 On-Line Documents. available in http://grouper.ieee.org/groups/1159/3/docs.html.

Pereira, D.C. (2002). Análise dinâmica por wavelets em um sistema com friç̧ão seca e amortecimento.

Ray, P.K., Dubey, H.C., Mohanty, S.R., Kishor, N., and Ganesh, K. (2010). Power quality disturbance detection in grid-connected wind energy system using wavelet and s-transform. In 2010 International Conference on Power, Control and Embedded Systems, 1-4. doi:10. 1109/ICPCES.2010.5698664.

Weeks, M. (2010). Digital signal processing using $M A-$ $T L A B \&$ wavelets. Jones \& Bartlett Learning.

Wilkinson, W.A. and Cox, M.D. (1996). Discrete wavelet analysis of power system transients. IEEE Transactions on Power Systems, 11(4), 2038-2044. doi:10.1109/59. 544682 . 